or low BMI $\left(<25 \mathrm{~kg} / \mathrm{m}^{2}\right)$. A marked difference was found in the time from diagnosis to the first surgical intervention between overweight and underweight $\left(\mathrm{BMI}<18.5 \mathrm{~kg} / \mathrm{m}^{2}\right)$ patients24 months versus 252 months, respectively $(P=0.043)$. The difference in time from diagnosis to the first surgical intervention between overweight patients and normal-weight patients $\left(\mathrm{BMl}<25 \mathrm{~kg} / \mathrm{m}^{2}\right)$, however, was not significant.

In the light of these findings, the authors suggest that overweight individuals with Crohn's disease might need to be more closely monitored than those of normal weight and that they might benefit from more aggressive immunomodulation at an earlier stage.

Original article Hass DJ (2006) The impact of increased body mass index on the clinical course of Crohn's disease. Clin Gastroenterol Hepatol 4: 482-488

\section{Fewer headaches for patients undergoing treatment for anal fissure}

Topical nitroglycerin is a common treatment for anal fissure that reduces internal-sphincter tone and facilitates blood flow and healing. Previous studies have, however, shown variable efficacy and side effects (principally headache) of nitroglycerin in this setting. This variation might be caused, in part, by inaccurate placement and dosage of nitroglycerin associated with methods of administration (e.g. open tubular applicators or gloved fingers). Torrabadella and Salgado have compared the efficacy and safety of nitroglycerin administered either intraanally, via a controlled dose-delivery device (DoseRite $^{\mathrm{TM}}$, Origyn, Tustin, CA) plus sideopening applicator, or administered perianally via a gloved finger, in treatment-naive patients with anal fissure.

Consecutive patients were randomly allocated to receive $2.25 \mathrm{mg}$ nitroglycerin three times daily, either intra-anally $(n=10)$-with removal of excess nitroglycerin that leaked from the anal verge-or perianally $(n=12)$. Average duration of follow-up was 59.2 days and 36.4 days (intra-anal and perianal group, respectively; visits every $7-10$ days). Clinical outcome (including fissure healing, sustained pain reduction, and referral for internal anal sphincterotomy) did not differ significantly between the groups. Intra-anal treatment was, however, associated with markedly fewer headaches ( 1 of 10 intra-anally treated patients versus 10 of 12 perianally treated) and greater reductions in resting anal pressure than perianal treatment. Headaches were so severe in seven perianally treated patients that they crossed over to the other group; six reported a subsequent improvement in their headaches.

The authors note that this drug delivery device might be useful in other disorders that require topical anal treatment.

Original article Torrabadella L and Salgado G (2005) Controlled dose delivery in topical treatment of anal fissure: pilot study of a new paradigm. Dis Colon Rectum 49: 1-4

\section{Laparoscopic cecostomy button placement in the treatment of pediatric fecal incontinence}

Parentally administered transanal enemas are an effective treatment for chronic constipation and encopresis in children with anorectal anomalies or Hirschsprung's disease, but compliance with such intrusive regimens can wane as patients age. Appendicostomy via the Malone procedure can provide a route for selfadministered antegrade colonic enemas, but the technique has several drawbacks: catheter insertion through the stoma can be painful and appendicostomy precludes use of the appendix as a urinary stoma, which is commonly required in this patient group. Yagmurlu et al. have now shown that laparoscopic cecostomy button placement (LCBP) spares the appendix, avoids laparotomy, and is effective in managing pediatric fecal incontinence.

The team reviewed data on three children with Hirschsprung's disease and four with anorectal malformations, who underwent LCBP using the 'U-stitch' method. The mean operative time was $33 \mathrm{~min}$ and no intraoperative complications were reported. Telephone follow-up showed that five of the seven caregivers were satisfied with the LCBP and that one or two enemas per day were sufficient to prevent the children soiling accidentally; however, two children were reported to have accidental bowel movements several nights a week.

The authors conclude that LCBP is a reversible and simple means of managing fecal incontinence in children with anorectal anomalies or Hirschsprung's disease. Of particular importance for patients' self-esteem, they highlight that a cecostomy button provides a solution 